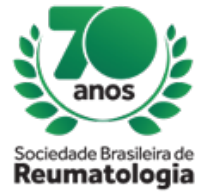

\title{
SEVERE AND TREATMENT RESISTANT UNDIFFERENTIATED SPONDYLOARTHRITIS
}

Pedro Otavio Rogowski (UNIOESTE, Francisco Beltrão, PR, Brasil), Luiz Felipe Becker (UNIOESTE, Francisco Beltrão, PR, Brasil), Tiago Osternack Malucelli (UNIOESTE, Francisco Beltrão, PR, Brasil)

\section{BACKGROUND}

Spondyloarthritis belong to a group of diseases that predominantly affects young men, which can cause arthritis of large peripheral and sacroiliac joints, as well as extra-articular manifestations such as uveitis, and cases that are refractory to treatment may evolve with inevitable sequelae.

\section{CASE REPORT}

Patient E.T.A.J., 15 years old, male, was initially attended in our public rheumatology clinic in April/2013 with oligoarticular arthritis (ankles and right knee), without axial complaints. He was a smoker (1.5 packs/year) and a regular practitioner of physical activities, with no relevant comorbidities or facts in previous history. Laboratory tests showed erythrocyte sedimentation rate (ESR) of $80 \mathrm{~mm}$, C-reactive protein (CRP) ++++, and positive HLA-B27, diagnosed as undifferentiated spondylarthritis, and it was not possible to use BASDAI. Treatment was initiated with right knee infiltration and nonsteroidal antiinflammatory drugs (NSAIDs), with little clinical response even with the exchange of NSAIDs, reason why in the following months the use of prednisone associated with methotrexate was started, keeping up with inflammatory activity tests. Due to the poor response to conventional disease modifying anti-rheumatic drugs (DMARDs), adalimumab (40 mg subcutaneously every 14 days) was started in October/2014, also with little response. In July/2015, adalimumab was replaced by golimumab, and the patient remained with oligoarthritis, ESR $121 \mathrm{~mm}$ and CRP 192. It evolved with acute right-sided uveitis, and golimumab was replaced by infliximab 300 mg every 8 weeks with increased doses of prednisone, but unfortunately evolved with amaurosis. In March/2016 a 60\% improvement was noted, but by mid 2017, there was a need to increase infliximab to $400 \mathrm{mg}$ every 6 weeks due to persistent arthritis. Thereafter, there was progressive improvement of the inflammatory picture (both clinical and laboratory), with a gradual reduction of corticotherapy. Since April/2018, the patient is clinically stable, with normalization of inflammatory activity (ESR and CRP negative) without corticosteroid therapy, using infliximab 400mg every 6 weeks, Sulfasalazine 3g/day and methotrexate $25 \mathrm{mg} /$ week. However, with permanent ocular sequelae and partial functional loss due to secondary arthrosis of the right knee and ankles, with associated thigh hypotrophy.

\section{CONCLUSION}

This case shows the therapeutic complexity that some refractory cases of spondyloarthritis require, and how the scheduling dynamics in a public clinic can influence the clinical outcome. Thus, we conclude that it is of fundamental importance the early reassessment and exchange/association of immunosuppressants according to protocol so that we avoid as much sequelae as our patient. 